\title{
SANTÉ MENTALE, ALCOOL/DROGUES ET VIOLENCE. ADAPTATION DU MODÈLE TRIPARTITE DE GOLDSTEIN
}

\author{
Perrine Poullot et al. \\ De Boeck Supérieur | Psychotropes
}

2008/3 - Vol. 14

pages 157 à 178

ISSN 1245-2092

Article disponible en ligne à l'adresse:

http://www.cairn.info/revue-psychotropes-2008-3-page-157.htm

Pour citer cet article :

Poullot Perrineet al., "Santé mentale, alcool/drogues et violence. Adaptation du modèle tripartite de Goldstein », Psychotropes, 2008/3 Vol. 14, p. 157-178. DOI : 10.3917/psyt.143.0157

Distribution électronique Cairn.info pour De Boeck Supérieur.

(c) De Boeck Supérieur. Tous droits réservés pour tous pays.

La reproduction ou représentation de cet article, notamment par photocopie, n'est autorisée que dans les limites des conditions générales d'utilisation du site ou, le cas échéant, des conditions générales de la licence souscrite par votre établissement. Toute autre reproduction ou représentation, en tout ou partie, sous quelque forme et de quelque manière que ce soit, est interdite sauf accord préalable et écrit de l'éditeur, en dehors des cas prévus par la législation en vigueur en France. II est précisé que son stockage dans une base de données est également interdit. 


\title{
Santé mentale, alcool/drogues et violence. Adaptation du modèle tripartite de Goldstein
}

\section{Mental health problems, substance abuse and violent criminal behavior. An adaptation of Goldstein's tripartite model}

\author{
Perrine Poullot \\ Candidate au doctorat en psychologie à l'Université de Montréal \\ Montréal, Québec, Canada \\ Courriel : perrinepoullot@hotmail.com \\ Denis Lafortune \\ Professeur agrégé à l'École de criminologie \\ de l'Université de Montréal \\ Serge Brochu \\ Professeur, École de criminologie de l'Université de Montréal \\ et codirecteur du RISQ
}

\begin{abstract}
Résumé : Plusieurs recherches démontrent que le fait de présenter un trouble mental sévère et un trouble concomitant lié à une substance serait associé à un risque plus élevé de comportements violents. Toutefois, il n'existe aucun modèle conceptuel expliquant la relation entre ce type de comorbidité psychiatrique et la violence. Cet article propose une première explication du phénomène basée sur une adaptation des volets psychopharmacologique et économicocompulsif du modèle tripartite de Goldstein, à la population atteinte d'un trouble mental sévère et persistant (la schizophrénie ou la maladie bipolaire) et d'un trouble concomitant lié à une substance.
\end{abstract}


Abstract: Many studies have shown an increased risk of violent behavior in people who present a severe mental disorder combined with a substance abuse problem. However researchers have yet to place this relationship into a conceptual framework. The following study suggest such a theoritical explanation based on Goldstein's (1985) economic compulsive and psychopharmacological models.

Mots clés : schizophrénie, trouble bipolaire, abus, dépendance, comorbidité, violence, modèle.

Keywords: schizophrenia, bipolar disorder, abus, drug dependence, comorbidity, violence, model.

Plusieurs recherches démontrent que le fait de présenter un trouble mental sévère et un trouble concomitant lié à une substance serait associé à un risque plus élevé de comportements violents (Côté \& Hodgins, 1990 ; Rice \& Harris, 1995 ; Swanson et al., 2002 ; Steadman et al., 1998 ; Fulwiler et al., 1997). Entre autres, Steadman et ses collègues (1998) ont observé que la présence d'un trouble lié à une substance augmentait significativement le taux de violence chez les individus relâchés d'établissements pour personnes atteintes de troubles mentaux sévères. Fulwiler $e t$ al. (1997) ont noté des résultats similaires, observant que la présence d'un trouble lié à une substance était un important facteur de risque de violence chez les gens atteints d'une maladie mentale sévère et chronique. Et, plus récemment, Swanson et al. (2002) ont identifié certaines variables reliant la maladie mentale sévère et la violence, parmi lesquelles figurait la présence d'un trouble lié à une substance.

La relation entre ce type de comorbidité psychiatrique et la violence est d'une importance non négligeable puisque de nombreuses études démontrent que les personnes atteintes de troubles mentaux sévères et persistants, tels la schizophrénie ou le trouble bipolaire, présentent un risque plus élevé de manifester un trouble lié à une substance que la population en général (Cuffel, 1996 ; Regier et al., 1990). Une étude américaine, souvent mentionnée à ce sujet, celle du Epidemiologic Catchment Area, démontre que le taux de prévalence à vie d'un trouble lié à une substance chez la population en général est de $17 \%$, alors qu'il est de $48 \%$ chez les individus atteints de schizophrénie et de $56 \%$ chez ceux atteints du trouble bipolaire (Regier et al., 1990 ; Kessler et al., 1996). Mercier et Beaucage (1997) ont observé des résultats similaires à l'aide d'une recension d'étu- 
des épidémiologiques américaines. Ils rapportent que les schizophrènes présentent une prévalence à vie d'un trouble d'abus de substances de $34 \%$ pour l'alcool et de $28 \%$ pour les drogues, et les patients bipolaires, une prévalence à vie de $46 \%$ pour l'alcool et de $41 \%$ pour les drogues.

Il n'existe aucun modèle conceptuel expliquant la relation entre ce type de comorbidité psychiatrique et la violence. Cependant, certains auteurs ont suggéré différents modèles expliquant la relation entre les troubles liés à une substance et la violence. Un de ces derniers, Goldstein, fit un premier effort d'intégration en regroupant sous un même modèle, trois conceptualisations unidimensionnelles : le modèle psychopharmacologique, selon lequel la violence découlerait des effets psychopharmacologiques de la substance ; le modèle économico-compulsif, selon lequel la violence résulterait de la nécessité d'obtenir de l'argent pour assouvir leur dépendance à une substance coûteuse ; et le modèle systémique, selon lequel la violence serait intrinsèque au réseau d'approvisionnement et de distribution de la drogue (Brochu, 2006). Ces derniers décrivent ainsi diverses formes de violence associées à différents types d'usage de drogues, à plusieurs contextes sociaux et à de multiples motivations derrière le passage à l'acte (Goldstein, 1985).

Le texte suivant constitue une première tentative d'adaptation des volets psychopharmacologique et économico-compulsif du modèle tripartite de Goldstein, à la population atteinte d'un trouble mental sévère et persistant (la schizophrénie ou la maladie bipolaire) et d'un trouble concomitant lié à une substance. Puisque le volet systémique décrit plutôt une violence résultant d'une implication dans le commerce illégal de la drogue (par exemple, les disputes de territoires entre des vendeurs de drogues, les règlements de compte, etc.) et non pas à un effet de la prise d'une substance, il ne répond pas aux propos du travail et ne sera pas, par conséquent, adapté à cette nouvelle population. En effet, Pernanen, Cousineau, Brochu et Sun (2002) expliquent que la violence décrite par le volet systémique ne constitue pas une conséquence directe de la consommation, mais de sa prohibition, décrivant alors davantage une relation indirecte drogue-violence, où il serait possible de substituer les drogues illicites à d'autres marchandises ou marchés illégaux.

Avant de débuter l'adaptation des volets psychopharmacologique et économico-compulsif de Goldstein, mentionnons, finalement, que les troubles mentaux, la médication psychotrope et les substances intoxicantes agissent tous au niveau des mêmes ensembles neuronaux du cerveau, entraînant des effets inévitables de synergie qui affectent l'état mental (Nadeau, 2001). Nous savons peu sur la nature de ces effets et la façon 
dont ils se manifestent, sinon que les effets de ces substances ne sont pas les mêmes chez une population aux prises avec des maladies mentales sévères et chroniques que chez la population en général. Les lecteurs sont donc avisés de tenir compte de ces limites, ce texte ayant été rédigé d'après les connaissances actuelles sur le phénomène.

\section{Le volet psychopharmacologique}

Le volet psychopharmacologique du modèle de Goldstein suggère que certains individus deviennent excitables, irrationnels et manifestent des comportements violents suite à une simple consommation ou à une consommation régulière de certaines substances spécifiques, soit, entre autres, l'alcool, les stimulants, les barbituriques et la phéncyclidine (PCP). Les comportements violents, auxquels ce volet fait référence, découleraient directement des effets psychopharmacologiques induits par la substance consommée, lors d'un état d'intoxication ou d'une période de sevrage (tels la désinhibition, l'appauvrissement du contrôle de soi, la détérioration du jugement, la formation d'idées paranoïdes ou d'un sentiment d'omnipotence, l'irritabilité et la montée d'impulsivité (Brochu, 2006)). Ainsi, selon ce volet, le fait d'avoir consommé une substance psychoactive aurait entraîné un comportement violent qui n'aurait probablement pas eu lieu autrement (Goldstein, 1985 ; Brochu, 1995).

\section{Le volet psychopharmacologique adapté à la population atteinte d'un trouble mental sévère et persistant et d'un trouble concomitant lié à une substance}

Afin de permettre l'adaptation du volet psychopharmacologique à la population présentant un trouble mental sévère et persistant et un trouble concomitant lié à une substance, rappelons ce que les études révèlent à propos :

a) des caractéristiques des troubles liés à une substance chez la population atteinte d'un trouble mental sévère ;

b) du niveau de sensibilité de cette population aux substances psychoactives ;

c) des effets de la médication psychotrope sur les troubles liés à une substance ;

d) et finalement de l'influence des substances psychoactives sur les symptômes psychotiques. 


\section{Caractéristiques des troubles liés à une substance chez la population atteinte d'un trouble mental sévère}

Contrairement à ce que propose l'hypothèse d'automédication (Khantzian,1985, 1997) voulant que les individus aux prises avec des troubles psychiatriques consomment dans le but de diminuer certains symptômes de leur maladie, Drake et Mueser (2000) rapportent, suite à une recension des écrits scientifiques, que les troubles liés aux substances, chez cette population, présentent les mêmes caractéristiques que ceux chez la population régulière, même en ce qui concerne les raisons pour lesquelles les individus des deux groupes consomment.

Tout d'abord, il a été démontré que, chez les deux groupes d'individus, l'alcool est la substance la plus consommée, suivi du cannabis et de la cocaïne (Mueser et al., 1992 ; Barry et al., 1995 ; Lehman et al., 1996 ; Brunette, Noordsy \& Green, 2005) ; ensuite, la consommation de substances psychoactives semble être un comportement social associé à des problèmes de désinhibition et d'instabilité psychosociale chez les deux groupes (Alverson, Alverson \& Drake, 2001) ; finalement, les gens atteints d'un trouble mental sévère disent consommer principalement pour les mêmes raisons que les toxicomanes non atteints d'une maladie mentale sévère (Dixon et al., 1991 ; Warner et al., 1994 ; Mueser, Drake \& Wallach, 1998), soit afin de combattre la solitude, l'anxiété sociale, l'ennui et l'insomnie, plutôt que de diminuer les symptômes spécifiques de leur maladie.

Warner et al. (1994) expliquent que, si ces individus consommaient réellement dans un but d'automédication, nous observerions une tendance générale à consommer une substance spécifique afin de calmer un symptôme spécifique d'une certaine maladie mentale. Cependant, les études empiriques ne démontrent pas un tel lien (Mueser, Drake \& Wallach, 1998 ; Dixon, 1999 ; Scheller-Gilkey et al., 2003).

\section{Sensibilité de la population atteinte d'un trouble mental sévère et persistant aux substances psychoactives}

Quoique les troubles liés aux substances présentent des caractéristiques semblables chez les deux groupes d'individus, Mueser, Drake et Wallach (1998), en se basant sur une recension des écrits et leurs propres analyses, observent que les deux groupes diffèrent sur un aspect central : la population atteinte d'un trouble concomitant sévère et persistant semble démontrer une sensibilité plus élevée aux effets causés par les substances psychoactives que la population régulière. En effet, des études révèlent 
que de faibles doses d'alcool ou d'autres substances entraînent chez les patients schizophrènes des conséquences défavorables, telles une exacerbation de la maladie et des difficultés au niveau du comportement, des relations et des finances (Dixon et al., 1990 ; Noordsy, 1991 ; Drake \& Mueser, 2000).

De plus, cette sensibilité plus élevée permettrait à ces individus de consommer de plus légères quantités de substances psychoactives. Lehman, Myers, Dixon, et Johnson (1994) s'aperçoivent en effet que les patients schizophrènes consomment de plus petites doses de drogues que les patients atteints d'un trouble de personnalité. Puisqu'ils consomment de plus petites doses, les individus atteints de troubles mentaux sévères ont moins de probabilité de développer une dépendance physique à ces substances (Drake et al., 1990 ; Mueser, Drake \& Wallach,1998), car celles-ci nécessitent un usage continu de doses élevées. En effet, Arias Horcajadas et ses collègues (1997) observent, chez un groupe de schizophrènes d'une clinique externe, un taux plus élevé d'abus d'alcool, de cocaïne, de cannabis et de nicotine que de dépendance à ces substances.

\section{Effets de la médication psychotrope sur les troubles liés à une substance chez la population atteinte d'une maladie mentale sévère et persistante}

De nombreuses études observent que la non-observance de la médication psychotrope constitue un phénomène très fréquent chez la population atteinte à la fois d'un trouble mental sévère et persistant et d'un trouble lié à une substance (Miner et al., 1997 ; Swartz et al., 1998 ; Osher \& Drake, 1996 ; Owen, Fischer, Booth \& Cuffel, 1996 ; Valenstein et al., 2006 ; Sajatovic, Valenstein, Blow, Ganczy \& Ignacio, 2006). Owen, Fisher, Booth et Cuffel (1996) ont observé, par exemple, que la présence d'un trouble lié à une substance était fortement associée à la non-observance de la médication chez les schizophrènes.

Plus récemment, Valenstein et ses collègues (2006) ont rapporté que le taux de schizophrènes présentant une faible observance de leur médication s'élevait jusqu'à $40 \%$. Le même phénomène a été observé chez les patients bipolaires (Goldberg et al., 1999 ; Sajatovic, Valenstein, Blow, Ganczy \& Ignacio, 2006).

Par contre, peu d'études se sont penchées sur l'interaction entre l'abus de substance et la réponse au traitement pharmacologique lorsqu'il y a observance de la médication. Celles qui ont été réalisées démontrent que, chez les schizophrènes, les antipsychotiques, à l'exception de cer- 
tains antipsychotiques atypiques tels la clozapine, la quétiapine ou la rispéridone, n'ont pas d'effets atténuants sur les troubles liés aux substances et peuvent, au contraire, les précipiter ou les exacerber (Buckley, 1998 ; Scheller-Gilkey et al., 2003 ; Voruganti, Heslegrave \& Awad, 1997 ; Zimmet et al., 2000 ; Green, 2005 ; Stuyt, Sabjbel \& Allen, 2006). En effet, certains antipsychotiques entraînent chez ces patients des effets secondaires désagréables, tels une dysphorie neuroleptique, qu'ils tenteraient d'atténuer à l'aide de substances psychoactives.

Les troubles liés aux substances découleraient donc, selon certains auteurs, dont Potvin, Stip et Roy (2003) et Voruganti, Heslegrave et Awad (1997), non pas d'un désir de diminuer certains symptômes positifs de la maladie, mais plutôt d'une envie de soulager certains symptômes négatifs, ainsi que les effets secondaires entraînés par la médication. Par exemple, une étude de Voruganti, Heslegrave et Awad (1997) révèle que, parmi un groupe de patients, quatre fois plus de sujets qui avaient manifesté un sentiment de dysphorie neuroleptique, suite à un traitement pharmacologique, avaient développé un trouble lié à une substance. Potvin, Stip et Roy (2003) suggèrent que l'effet bénéfique de la clozapine sur les troubles liés à une substance serait lié à la diminution des symptômes négatifs causés par la maladie, sans pour autant entraîner des effets extrapyramidaux. Ceci expliquerait, d'une part, pourquoi, sur un échantillon de 58 schizophrènes présentant un trouble lié à une substance, Zimmet et al. (2000) ont observé que $85 \%$ d'entre eux avaient diminué leur consommation après avoir débuté un traitement à la clozapine. Dans le même sens, Brunette, Drake, Xie, McHugo et Green (2006) ont noté moins de rechutes chez ceux ayant cessé leur consommation suite à l'application d'un tel traitement.

Bien que la clozapine soit pour l'instant l'antipsychotique atypique le plus souvent associé à une stabilisation de l'état mental, à une diminution des états de besoin ${ }^{1}$ de cocaïne et à une réduction de la consommation de substances intoxicantes chez les individus présentant à la fois un trouble sévère et persistant et un trouble lié à une substance, d' autres antipsychotiques atypiques tels que la quétiapine, l'olanzapine ou la rispéridone se sont également révélés efficaces dans le traitement de cette double problématique et mériteraient, par conséquent, d'être étudiés davantage (Noordsy, O'Keefe, Mueser \& Xie, 2001 ; Smelson, Losonczy, Davis, Kaune, Williams \& Ziedonis, 2002 ; Brown, Netjek, Perantie \& Bobadilla, 2002 ; Green, 2006 ; Stuyt, Sabjel \& Allen, 2006).

1. « Craving ». Notre traduction. 


\section{L'influence des substances psychoactives sur les symptômes psychotiques}

Brunette, Mueser, Xie et Drake (1997) ont révisé différents types d'études ayant tenté d'explorer la relation entre l'abus de substances psychoactives et les symptômes psychotiques auprès de patients schizophrènes ou schizoaffectifs. Les études effectuées en laboratoire démontrent que les hallucinogènes et les amphétamines augmentent parfois les symptômes positifs psychotiques. Les études corrélationnelles et longitudinales montrent que l'usage de la cocaïne et du cannabis est souvent associé à une exacerbation des symptômes positifs et que l'alcool et la cocaïne engendrent souvent une augmentation des symptômes dépressifs. Les résultats ne sont pas consistants puisque la moitié des études affirment qu'il existe une relation entre l'abus de substances et la présence de symptômes plus aigus, alors que l'autre moitié n'observe aucune relation ${ }^{2}$.

Plus récemment, Talamo, Centorrino, Tondo, Dimitri, Hennen et Baldessarini (2006) ont combiné les résultats d'une série d'études indépendantes ayant comparé les taux de symptômes positifs et négatifs rapportés par des schizophrènes atteints d'un trouble lié à une substance et par ceux ne présentant pas une telle comorbidité. Cette analyse leur a permis d'observer une plus grande importance de symptômes positifs et une plus faible présence de symptômes négatifs chez le premier groupe que chez le second. Notons cependant que cette étude ne permet pas d'établir une relation de cause à effet entre ces résultats et la présence d'un trouble lié à une substance, celle-ci pouvant être la cause ou la conséquence d'une plus grande importance de symptômes positifs.

À partir des résultats obtenus par ces études, il est possible de suggérer quelques hypothèses permettant d'adapter le volet psychopharmacologique à la population d'individus présentant à la fois un trouble mental sévère et persistant et un trouble lié à une substance.

Premièrement, le fait que le type de drogue consommé soit associé à une désinhibition et à une instabilité psychosociale chez cette population, comme chez la population en général, que les effets recherchés par les consommateurs soient également les mêmes, et puisque les troubles liés aux substances présentent les mêmes caractéristiques chez les deux groupes, il est possible de se demander pourquoi la présence de ces troubles ne serait pas associée à la violence chez les individus atteints de trou-

2. Voir Brunette, Mueser, Xie et Drake (1997) pour une synthèse détaillée des études recensées par ces derniers. 
bles mentaux sévères, si elle l'a été chez la population régulière (Brochu, 1994, 1995, 2006).

Deuxièmement, si nous considérons que la population aux prises avec un trouble mental sévère démontre une sensibilité plus élevée aux effets de ces substances, réagissant ainsi plus fortement à de plus petites doses, il est possible de croire que les mécanismes responsables de la violence entraînés par la consommation d'alcool ou de drogues (désinhibition, appauvrissement du contrôle de soi, détérioration du jugement, formation d'idées paranoïdes ou d'un sentiment d'omnipotence, irritabilité et montée d'impulsivité (Brochu, 2006)) apparaîtraient alors avec une fréquence et une intensité similaires, ou même supérieures, chez cette population.

Troisièmement, cette sensibilité plus élevée, causant une exacerbation de la maladie, augmente davantage les risques de violence chez cette population qui, par ailleurs, selon certains auteurs, présente déjà un risque plus élevé de violence que la population régulière (Link \& Stueve, 1994 ; Swanson, Borum, Swartz \& Monahan, 1996; Rice \& Harris, 1995; Monahan \& Appelbaum, 2000 ; Tiihonen et al., 1997 ; Brennan, Mednick \& Hodgins, 2000 ; Arseneault, Moffitt \& Caspi, 2000 ; Feldmann, 2001). En effet, Monahan et Appelbaum (2000) ont observé un risque de violence de $15 \%$ chez les gens atteints de maladie bipolaire et de 17,5\% chez les gens atteints d'un trouble psychotique. Même si ces taux sont moins élevés que celui présenté par les gens atteints d'un trouble lié à une substance $(29 \%)$, ils seraient tout de même plus élevés que celui de la population régulière, comme le démontrent plusieurs études telles celles de Tiihonen et al. (1997) ou de Feldmann et al. (2001). En effet, Tiihonen et al. (1997) observent un risque sept fois plus élevé de violence chez les schizophrènes que chez la population régulière et Feldmann et al. (2001) constatent que le risque de violence est aussi plus élevé chez les gens atteints de maladie bipolaire, particulièrement lorsque ces derniers sont en phase maniaque. Finalement, une étude d'Arseneault, Moffitt et Caspi (2003) démontre que, parmi un échantillon de 956 jeunes adultes de 21 ans, ceux qui présentaient un trouble du spectre de la schizophrénie rapportaient, dans la dernière année, des taux plus élevés de vols, de batailles de gang et d'assauts envers les gens, comparativement aux autres.

Selon certains auteurs, cette association entre les troubles mentaux sévères et la violence serait due à la présence de certains symptômes positifs plus spécifiquement liés à un risque plus élevé de comportements violents (Link \& Stueve, 1994 ; Hodgins, Côté \& Toupin, 1998 ; Swanson, Borum, Swartz \& Monahan, 1996 ; Link, Stueve \& Phelan, 1998). 
Ainsi, Link et Stueve (1994) ont identifié certains symptômes potentiellement reliés à la violence, tels "l'impression que les gens souhaitent vous faire du mal » 3 , « l'impression que votre cerveau est dominé par des forces hors de notre contrôle » 4 , etc., qu'ils ont nommés «threat/control override symptoms $\gg 5$ (TCO). À l'aide d'une analyse, ces auteurs ont démontré que seuls ces symptômes étaient statistiquement reliés à la violence. Il est donc possible de croire que l'exacerbation de la maladie entrânée par la consommation d'une substance psychoactive augmente les risques de violence chez cette population car les symptômes positifs, associés à un risque de comportement violent, sont ainsi accentués. En effet, une étude de Pristach et Smith (1996) révèle que des patients schizophrènes rapportaient une exacerbation de leurs symptômes d'hallucination et de paranoïa, donc des symptômes TCO, suite à une consommation d'alcool.

Quatrièmement, comme certaines études démontrent que la nonobservance est un phénomène fréquemment observé chez les individus atteints d'un trouble mental sévère et d'un trouble concomitant lié à une substance (Miner et al., 1997 ; Swartz et al., 1998 ; Osher \& Drake, 1996 ; Owen, Fischer, Booth \& Cuffel, 1996), la médication qui aurait pu agir à titre de facteur de protection contre l'exacerbation de la maladie entrấnée par la consommation de substances psychoactives, donc contre les risques de violence qui s'y rattachent, est plutôt mise de côté. Ceci explique sûrement pourquoi Swartz et ses collègues (1998) ont observé, lors d'une étude réalisée auprès de 331 patients atteints d'un trouble mental sévère, que la combinaison d'un trouble de consommation et d'une nonobservance de la médication était associée à un comportement violent grave (soit une agression à main armée, soit une agression entraînant une blessure) manifesté lors des quatre mois précédant l'hospitalisation. Par ailleurs, lorsqu'il y a observance de la médication chez ces patients, les recherches mentionnées plus haut démontrent que certains médicaments psychotropes précipitent les troubles liés aux substances, entraînant une fois de plus les risques de violence qui s'y rattachent.

Une combinaison de tous ces facteurs expliquerait probablement pourquoi plus de 100 études démontrent, en effet, que cette comorbidité psychiatrique est associée à de nombreuses conséquences négatives, dont la violence, des problèmes avec la justice et l'incarcération (Bartels, Drake, Wallach \& Freeman, 1991 ; Cuffel et al., 1994 ; Swanson, Holzer, Ganju

3. « That there were people who wished to do you harm ». Notre traduction.

4. «That your mind was dominated by forces beyond your control». Notre traduction.

5. « Symptômes de menace et/ou de perte de contrôle ». Notre traduction. 
\& Jono, 1990 ; Abram \& Teplin, 1991 ; Drake \& Mueser, 2000). Lorsque les effets de l'alcool et des autres drogues agissent sur des personnes déjà aux prises avec des difficultés cognitives, comportementales, sociales et économiques, le résultat menace d'être particulièrement négatif (Brunette, Mueser, Xie \& Drake, 1997).

Ainsi, comme il est possible de l'observer à l'aide de la figure 1, trois facteurs reliés à la présence d'une maladie mentale sévère et persistante, seuls ou combinés, peuvent mener l'individu à consommer une substance psychoactive, à savoir : a) sa vulnérabilité face à son trouble psychique grave, b) les symptômes négatifs de sa maladie et c) les effets désagréables de sa médication psychotrope qu'il souhaite annihiler. D'autre part, la sensibilité plus élevée de ces individus provoque une réaction plus forte à l'usage de la drogue, qui, combinée ou non à une médication psychotrope, entraîne un état d'intoxication, donc une altération de la neurotransmission. Cet état engendre, à son tour, les mécanismes responsables de la violence associés à la consommation d'alcool ou de drogues, les symptômes TCO liés eux aussi à la violence, ainsi que les risques de nonobservance de la médication. Le résultat final serait une augmentation des risques de violence.

Figure 1 : Présentation schématique du modèle psychopharmacologique adapté à la population d'individus présentant un trouble mental sévère et persistant et un trouble concomitant lié à une substance

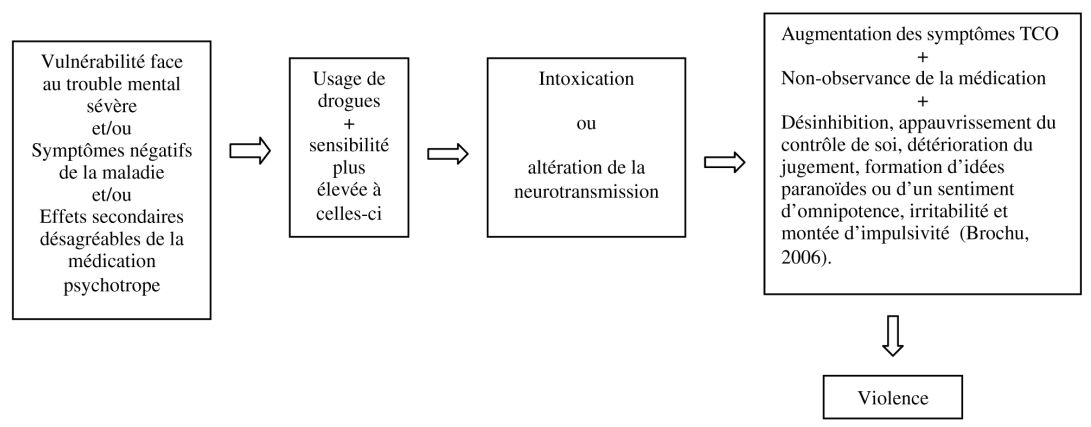

\section{Le volet économico-compulsif}

Le volet économico-compulsif du modèle de Goldstein (1985) suggère que certains individus commettent des crimes violents pour monnayer l'achat de leurs substances (ex. : le vol à main armée). Les individus faisant partie de cette catégorie ne sont pas motivés principalement par une envie d'agir violemment, mais plutôt par un désir d'obtenir de l'argent pour 
acheter des drogues coûteuses, telles l'héroïne ou la cocaïne, qui sont très souvent consommées de façon compulsive. C'est pourquoi les moyens violents sont de préférence évités.

\section{Le volet économico-compulsif adapté à la population atteinte d'un trouble mental sévère et persistant et d'un trouble concomitant lié à une substance}

Brochu (2006) spécifie que le volet économico-compulsif s’appliquerait spécialement aux individus :

- qui ont un revenu limité ;

- $\quad$ et qui sont fortement dépendants de drogues coûteuses.

Analysons ces deux éléments pour la population à l'étude.

\section{Statut socio-économique de la population atteinte d'un trouble mental sévère et persistant et d'un trouble concomitant lié à une substance}

Il est reconnu que la schizophrénie est associée à des déficits au niveau cognitif et social (Blanchard \& Neale, 1994); cependant les déficits associés à la maladie bipolaire ne sont pas aussi clairement établis (Goldberg, 1999 ; Dickerson, Sommerville, Origoni, Ringel \& Parente, 2001). Certaines études démontrent que, lorsqu'on les compare aux schizophrènes, les individus atteints de la maladie bipolaire présentent des difficultés similaires au niveau social (Grossman, 1991), de même qu'au niveau cognitif (Albus et al., 1996 ; Addington \& Addington, 1997).

Ainsi, les gens atteints de troubles mentaux sévères ont en général un faible niveau d'éducation (Kessler, Foster, Saunders \& Stang, 1995), un manque d'habiletés professionnelles (Alverson, Alverson \& Drake, 2001) et ils sont, par conséquent, très souvent sans emploi (Jablensky et al., 1999 ; Mueser, Salyers \& Mueser, 2001 ; Mulkern \& Manderscheid, 1989). En effet, de nombreuses études observent que les difficultés que présentent ces personnes contribuent directement au faible taux d'employabilité (McGurk \& Meltzer, 2000). Par exemple, McGurk et Mueser (2003) ont observé que, parmi un échantillon d'individus atteints d'une maladie mentale grave, ceux qui étaient sans emploi démontraient de plus grandes difficultés cognitives, ainsi que des symptômes plus prononcés (positifs et négatifs) de la maladie.

Comme il a été mentionné, non seulement ces individus présentent des difficultés cognitives, mais également des difficultés sociales qui, 
d'une part, nuisent à leur performance au travail ou à leur recherche d'emploi, et qui interfèrent également dans leurs relations familiales et sociales (Bellack, 1997). Ces difficultés associées à la maladie, et le faible taux d'employabilité qui en résulte, poussent certains individus vers la pauvreté et l'isolation, les empêchant de retourner à leur niveau de fonctionnement prémorbide (Fox, 1990 ; Maneros et al., 1992).

En effet, Melle, Friis, Hauff et Vaglum (2000) ont voulu observer le niveau d'intégration de 81 schizophrènes sept ans après avoir reçu leur congé d'un hôpital psychiatrique. Ils ont constaté que 47 \% étaient isolés socialement et que $94 \%$ étaient sans emploi. Il n'est donc pas surprenant que Robins et Regier (1990) observent que la schizophrénie se rencontre chez $8,7 \%$ des sans abris, alors qu'elle ne touche que $1 \%$ de la population en général. Lorsqu'il s'agit d'individus à la fois atteints d'une maladie mentale sévère et persistante et d'un trouble concomitant lié à une substance, les recherches démontrent que ces personnes souffrent aussi de pauvreté, d'un faible niveau d'éducation, d'un faible support social, d'un manque d'emploi, d'un manque d'habiletés sociales, cognitives et professionnelles (Cuffel, 1996 ; Brunette, Mueser, Xie \& Drake, 1997).

\section{La dépendance aux substances et les états de besoin plus intenses ressentis par cette population}

Tel que mentionné, les individus atteints d'une maladie mentale sévère et persistante, démontrant une sensibilité plus élevée aux substances psychoactives, réagissent plus fortement à de plus faibles doses de celles-ci (Mueser, Drake \& Wallach, 1998). Par conséquent, ces individus consomment de plus faibles quantités de substances (Lehman, Myers, Dixon \& Johnson, 1994) et ont moins de probabilité, selon certains auteurs, de développer une dépendance physique à ces dernières, celle-ci nécessitant un usage continu de doses élevées (Drake et al., 1990 ; Mueser et al., 1998).

Des études appuyant cette tendance révèlent que 16 à $40 \%$ des schizophrènes abuseraient de la cocaïne (ce taux pouvant même grimper jusqu'à $70 \%$ dans certains milieux hospitaliers ou cliniques externes) (Regier et al., 1990 ; Ziedonis \& Trudeau, 1997), mais seulement $17 \%$ seraient dépendants de cette même substance (Carol, 2000). Cependant, il est important de mentionner que d'autres auteurs observent plutôt le phénomène inverse chez cette même population, soit des taux de dépendance supérieurs aux taux d'abus (Hodgins \& Côté, 2002 ; Grant et al., 2004). Ainsi, une étude réalisée par Hodgins et Côté (2002), auprès de patients atteints d'un trouble mental sévère et persistant, démontre que 
$37,5 \%$ de ces derniers consomment de la cocaïne, et qu'il s'agit, pour la majorité d'entre eux $(26,7 \%)$, d'une dépendance sévère. Grant et ses collègues (2004) ont observé le même phénomène, lors d'une étude américaine réalisée auprès d'un large échantillon d'individus vivant dans la communauté, soit des taux de dépendance aux substances plus élevés que les taux d'abus chez les gens présentant une maladie bipolaire. Par ailleurs, lorsqu'il y a dépendance à certaines de ces substances, telle la cocaïne, il a été observé que les gens atteints d'une maladie mentale sévère et persistante ressentent des états de besoin plus intenses que la population régulière, vu leur plus grande instabilité psychosociale et leur vulnérabilité neurobiologique (Carol, 2000 ; Carol, Smelson, Losonczy \& Ziedonis, 2001 ; Smelson et al., 2002).

À l'aide de ces recherches, il est possible de proposer une adaptation du volet économico-compulsif à la population atteinte à la fois d'un trouble mental sévère et d'un trouble concomitant lié à une substance. Les données recueillies nous démontrent que, non seulement les gens atteints d'un trouble mental sévère présentent des taux élevés de dépendance ou d'abus, qui sont d'ailleurs bien plus élevés que ceux observés chez la population régulière ou carcérale (Pernanen, Cousineau, Brochu \& Sun, 2002 ; Schneeberger, 1995), mais encore, pour une majorité d'entre eux, ils souffrent de pauvreté, répondant ainsi aux deux caractéristiques particulièrement associées à la violence lucrative.

En effet, les gens atteints d'une maladie mentale grave et chronique présentent de nombreux déficits au niveau cognitif, social et professionnel, et seule une minorité réussit à se faire engager et à maintenir un emploi (Hodgins \& Janson, 2002). Isolés et sans emploi, la plupart d'entre eux vivent dans la pauvreté. Il est facile de s'imaginer l'ampleur des difficultés financières auxquelles sont confrontés ceux qui, en plus, dépendent de substances coûteuses. N'ayant pas l'argent nécessaire pour subvenir aux dépenses usuelles qu'engendre une telle dépendance, ils se voient ainsi sûrement obligés, tout comme la population régulière aux prises avec un trouble de dépendance, d'employer tous les moyens qui leur sont disponibles pour se procurer cet argent.

Ces individus traverseraient ainsi les mêmes étapes que la majorité de la population régulière, en cherchant d'abord à réduire l'ensemble les dépenses, à augmenter les heures de travail, s'ils ont un emploi, à emprunter de l'argent, puis à vendre leur biens, pour finalement commettre des crimes lucratifs qui risquent de devenir de plus en plus violents, au fur et à mesure que le niveau de consommation de l'individu augmente (voler de l'argent aux proches jusqu'au vol à main armée, par exemple). En 
effet, plus la consommation de l'individu augmente, plus son désir d'obtenir de l'argent rapidement augmente. Il risque de devenir désespéré et, peu importe les conséquences que cela pourrait entraîner, ses crimes deviennent de plus en plus impulsifs et moins organisés (Parent \& Brochu, 2002).

Ainsi, Brochu et Cousineau (2003) rapportent qu'un détenu sur cinq affirme que le délit à l'origine de l'incarcération avait pour but de se procurer de la drogue. Comme les individus atteints à la fois d'un trouble sévère et d'un trouble lié à une substance commettent davantage de crimes violents que de crimes non violents (Hodgins, Lapalme \& Toupin, 1999) et comme ils présentent des difficultés cognitives, comportementales et sociales, il est possible de croire que ces derniers, entraînés par une forte motivation de se procurer de l'argent, agissent de façon encore plus impulsive que la population régulière, employant rapidement des moyens inorganisés, donc plus risqués. D'autant plus que certains auteurs observent que les schizophrènes dépendants à la cocaïne ressentent des états de besoin plus intenses que la population régulière (Carol, 2000; Carol, Smelson, Losonczy \& Ziedonis, 2001 ; Smelson et al., 2002), ce qui, on présume, les motiverait probablement davantage à passer à l'acte.

Comme il est résumé à la figure 2 , un taux élevé d'individus aux prises avec un trouble de santé mental sévère et persistant développent une dépendance à une substance. Puisque la majeure partie de cette population démontre déjà une pauvreté due à un manque d'emploi, un faible niveau d'éducation, un manque de support social, d'habiletés cognitives, sociales et professionnelles, une dépendance à une substance coûteuse, telle la cocaïne, augmenterait le besoin d'argent de ces derniers. Ce besoin d'argent, combiné au risque de violence associé à la maladie (symptômes TCO), augmenterait ainsi les risques de violence lucrative.

Figure 2 : Présentation schématique du modèle économico-compulsif adapté à la population d'individus présentant un trouble mental sévère et persistant et un trouble concomitant lié à une substance.

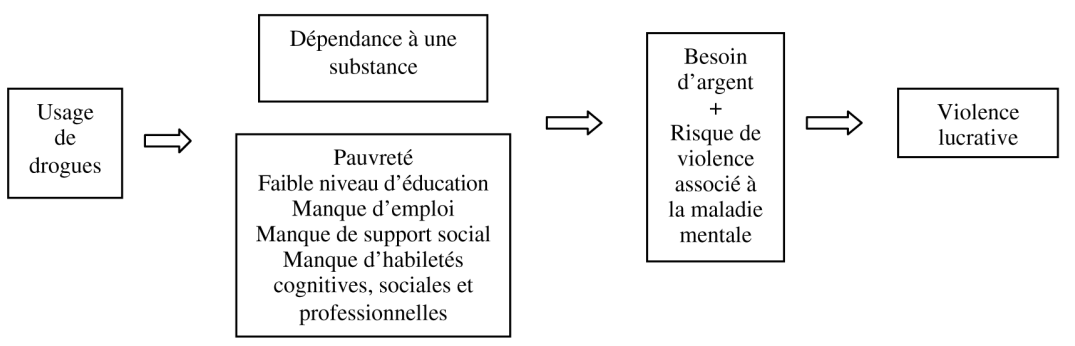




\section{Conclusion}

Le modèle tripartite de Goldstein (1985) décrit trois types de violence reliés aux troubles liés à une substance : la violence psychopharmacologique, la violence économico-compulsive et la violence systémique. Les études démontrent que, chez la population régulière, la majeure partie des homicides reliés à la drogue découlent du système de distribution établi pour pallier la prohibition de celle-ci. Quant à la violence directement liée à la consommation d'une substance, celle de nature lucrative serait davantage observée chez la population en général, que celle de nature psychopharmacologique. En effet, les cas de violence psychopharmacologique, à l'exception des actes violents accomplis sous l'influence de l'alcool, sont plutôt rares (Brochu, 1995).

Cependant, les études mentionnées auparavant appliquées à la population d'individus atteints d'un trouble mental sévère et persistant, telles la schizophrénie ou la maladie bipolaire, semblent appuyer davantage le volet psychopharmacologique, que le volet économico-compulsif, liant majoritairement la violence manifestée par ces individus à l'état d'intoxication, donc aux effets psychopharmacologiques de la substance psychoactive. L'incertitude face aux probabilités élevées ou faibles que présente cette population de développer une dépendance à une substance, ainsi que l'insuffisance des études consacrées à l'identification des motifs à l'origine des délits commis par celle-ci, limitent beaucoup notre compréhension de la violence lucrative manifestée par ces individus.

Davantage de recherches devraient ainsi être réalisées afin de pouvoir clairement évaluer l'importance de sa fréquence. Toutefois, que ces individus soient, ou non, moins à risque de développer un trouble de dépendance à une substance qu'un trouble d'abus, retenons simplement que des taux non négligeables de dépendance à la cocaïne ont tout de même été observés chez ces derniers qui, semble-t-il, seraient certainement responsables d'une partie de la violence manifestée. Finalement, bien que la majorité des cas de violence rapportés renvoient typiquement aux volets décrits plus haut, Goldstein précise que certains cas sont parfois le résultat d'une combinaison de ces derniers.

La violence manifestée par les individus présentant une maladie mentale sévère et un trouble concomitant lié à une substance est un phénomène qui intrigue plusieurs chercheurs (Côté \& Hodgins, 1990 ; Swanson, Holzer, Ganju \& Jono, 1990 ; Klassen \& O’Connor, 1988). Cependant, le manque d'information se rapportant à l'étiologie de cette comorbidité, à l'interaction entre ces deux problématiques ou à celle entre la prise de médication psychotrope et la consommation simultanée de substances psychoactives, de même que leurs influences sur les comportements vio- 
lents, limitent beaucoup notre compréhension du phénomène et ne nous permettent, pour l'instant, que de générer certaines hypothèses qu'il serait, toutefois, intéressant de vérifier empiriquement.

Article reçu en juin 2007 et accepté en août 2008

\section{Bibliographie}

Abram KM \& Teplin LA: "Co-occurring disorders among mentally ill jail detainees: Implications for public policy" - In American Psychologist, 46 (10) : 1036-1045 (1991)

Addington J \& Addington D: "Attentional vulnerability indicators in schizophrenia and bipolar disorder" - In Schizophrenia Research, 23 : 197-204 (1997)

Albus M, Hubmann W, Wahlhein C, Sobizack N, Franz U \& Mohr F: "Contrast in neuropsychological test profile between patients with first-episode schizophrenia and firstepisode affective disorders" - In Acta Psychiatrica Scandinavica, 94 : 87-93 (1996)

Alverson H, Alverson M, Drake RE: "Social patterns of substance-use among people with dual diagnosis" - In Mental Health Services Research, 3 (1) : 3-14 (2001)

Arias Horcajadas F, Padin Calo JJ \& Fernandez Gonzalez MA: "Drug use and dependence in schizophrenia" - In Actas Luso-Espanolas de Neurologia, Psiquiatria y Ciencias Afines, 25 (6) : 379-389 (1997)

Arseneault L, Moffitt TE, Caspi A, Taylor PJ \& Silva PA: "Mental disorders and violence in a total birth cohort: Results from the Dunedin Study" - In Archives of General Psychiatry, 57 (10) : 979-986 (2000)

Barry KL, Fleming, MF, Greenley J, Widlak P, Kropp S et McKee D: "Assessment of alcohol and other drug disorders in the seriously mentally ill" - In Schizophrenia Bulletin, 21 (2) : 313-321 (1995)

Bartels SJ, Drake RE, Wallach MA \& Freeman DH: "Characteristic hostility in schizophrenic outpatients" - In Schizophrenia Bulletin, 17 (1) :163-171 (1991)

Bellack AS: "Social skills deficits and social skills training: New developments and trends" - In HD Brenner, W Boeker, et al.: Towards a comprehensive therapy for schizophrenia (p. 137-146) (1997)

Blanchard JJ \& Neale JM: "The neuropsychological signature of schizophrenia: Generalized or differential deficit?" - In American Journal of Psychiatry, 151 (1) : 40-48 (1994)

Brennan PA, Mednick SA \& Hodgins S: "Major mental disorder and criminal violence in Danish birth cohort" - In Archives of General Psychiatry, 57 : 494-500 (2000)

Brochu S : Drogue et criminalité : Mythe ou réalité? - Montréal, Centre international de criminologie comparée (1994)

Brochu S : Drogue et criminalité : une relation complexe - Montréal, Presses de l'Université de Montréal (1995)

Brochu S \& Cousineau M-M : «Drogues et questions criminelles : un état de la question à partir d'études québécoises » - In LeBlanc M, Ouimet $\mathrm{M}$, Szabo $\mathrm{D}:$ Traité de criminologie empirique, 3e édition : 503-548, Montréal, Presses de l'Université de Montréal (2003).

Brown ES, Nejtek VA, Perantie DC \& Bobadilla L: "Quetiapine in bipolar disorder and cocaine dependence" - In Bipolar Disorders, 4 (6) : 406-11 (2002)

Brunette MF, Mueser KT, Xie H \& Drake RE: "Relationships between symptoms of schizophrenia and substance abuse" - In Journal of Nervous \& Mental Disease, 85 (1) : 13-20 (1997) 
Brunette MF, Noordsy DL \& Green AI: "Pharmacologic treatments for co-occuring substance use disorders in patients with schizophrenia : a research review" - In Journal of Dual Diagnosis, $1:$ 41-55 (2005)

Brunette M.F, Drake RE, Xie H, McHugo GJ \& Green AI: "Clozapine use and relapses of substance use disorder among patients with co-occurring schizophrenia and substance use disorders" - In Schizophrenia Bulletin, 32 (4) : 637-43 (2006)

Buckley PF: "Novel antipsychotic medications and the treatment of comorbid substance abuse in schizophrenia" - In Journal of Substance Abuse Treatment, 15 (2) : 113-116 (1998)

Carol G: "A comparision of cocaine craving, social support and Narcotics Anonymous involvement between schizophrenics and nonschizophrenics" - In Dissertation Abstracts International: Section B: the Sciences \& Engineering, 60, 12-B (2000)

Carol G, Smelson DA, Losonczy MF \& Ziedonis D: "Alcohol \& Drug Abuse: A preliminary investigation of cocaine craving among persons with and without schizophrenia" - In Psychiatric Services, 52 (8) : 1029-1031 (2001)

Côté G. \& Hodgins S: "Co-occurring mental disorders among criminal offenders. Bulletin of the American Academy of Psychiatry \& the Law, 18 (3) : 271-281 (1990)

Cuffel BJ: "Violent and destructive behavior among the severely mentally ill in rural areas: Evidence from Arkansas community mental health system" - In Community Mental Health Journal, 30 (5) : 495-504 (1994)

Cuffel BJ: "Comorbid substance use disorder : prevalence, patterns of use, and course" - In New Directions for Mental Health Services, 70 : 93-105 (1996)

Dickerson FB, Sommerville J, Origoni AE, Ringel NB \& Parente F: "Outpatients with schizophrenia and bipolar I disorder: Do they differ in their cognitive and social functioning?" - In Psychiatry Research, 102 (1) : 21-27 (2001)

Dixon L, Haas G, Weiden PJ, Sweeney J, et al. : "Drug abuse in schizophrenic patients: Clinical correlates and reasons for use" - In American Journal of Psychiatry, 148 (2) : 224-230 (1991)

Dixon L: "Dual diagnosis of substance abuse in schizophrenia: Prevalence and impact on outcomes" - In Schizophrenia Research, 35 (Suppl), s93-s100 (1999)

Drake RE, Osher FC, Noordsy DL, Hurlbut SC, et al. : "Diagnosis of alcohol use disorders in schizophrenia" - In Schizophrenia Bulletin, 16 (1) : 57-67 (1990)

Drake RE \& Mueser KT: "Psychosocial approaches to dual diagnosis" - In Schizophrenia Bulletin, 26 (1) : 105-118 (2000)

Feldmann TB: "Bipolar disorder and violence" - In Psychiatric Quarterly, 72 (2) : 119-129 (2001)

Fox JW: "Social class, mental illness, and social mobility: the social selection drift hypothesis for serious mental illness" - In Journal of Health and Social Behavior, $31: 344-353$ (1990)

Fulwiler C, Grossman H, Forbes C \& Ruthazer R: "Early-onset substance abuse and community violence by outpatients with chronic mental illness" - In Psychiatric Services 48 (9) : 1181-1185 (1997)

Goldberg JF, Garno JL, Leon AC, et al.: "A history of substance abuse complicates remission from acute mania in bipolar disorder" - In Journal of Clinical Psychiatry, $60: 733-740$ (1999)

Goldberg TE: "Some fairly obvious distinctions between schizophrenia and bipolar disorder" - In Schizophrenia Research, 39 : 127-132 (1999)

Goldstein PJ: "The drugs/violence nexus: A tripartite conceptual framework" - In Journal of Drug Issues, 15 (4) : 493-506 (1985) 
Grant BF, Stinson FS, Dawson DA, et al.: "Prevalence and co-occurrence of substance use disorders and independent mood and anxiety disorders: results from the National Epidemiologic Survey on Alcohol and Related Conditions" - In Archives of General Psychiatry, 6 : 807-816 (2004)

Green AI: "Schizophrenia and comorbide substance use disorder: effects of antipsychotics" - In Journal of Clinical Psychiatry, 66 (suppl 6) : 21-26 (2005)

Grossman LS, Harrow M, Goldberg JF \& Fichtner CG: "Outcome of schizoaffective disorder at two long-term follow-ups : comparison with outcomes of schizophrenia and affective disorder" - In American Journal of Psychiatry, 148 : 1359-1365 (1991)

Hamera E, Schneider JK \& Deviney S: "Alcohol, cannabis, nicotine, and caffeine use and symptom distress in schizophrenia" - In Journal of Nervous \& Mental Disease, 183 (9) : 559-565 (1995)

Hodgins S, Côté G \& Toupin J: "Major mental disorder and crime : An ethiological hypothesis" - In D Cooke, A Forth, RD Hare: Psychopathy:Theory, Research and Implications for Society, Dortrecht, Kluwer : 231-256 (1998)

Hodgins S, Lapalme M. \& Toupin J: "Criminal activities and substance use of patients with major affective disorders and schizophrenia: A 2-year follow-up" - In Journal of Affective Disorders, 55 (2-3) : 187-202 (1999)

Hodgins S: "The major mental disorders and crime: Stop debating and start treating and preventing" - In International Journal of Law and Psychiatry, 24 : 427-446 (2001)

Hodgins S. \& Janson C-G: Criminality and violence among the mentally disordered: The Stockholm Project Metropolitan - New York, Cambridge University Press (2002)

Hodgins S \& Côté G : «Les troubles mentaux et le comportement criminel »- In LeBlanc M, Ouimet M, Szabo D : Traité de criminologie empirique, 3e édition : 503548, Montréal, Presses de l'Université de Montréal (2003)

Jablensky A, McGrath J, Herrman H, Castle D, Gureje O, Morgan V \& Korten A: People living with psychotic illness: an Australian Study 1997-1998 - Canberra, Australia, Commonwealth of Australia (1999)

Kessler RC, Foster CL, Saunders WB\& Stang PE: "Social consequences of psychiatric disorders I: Educational attainment" - In American Journal of Psychiatry, 152 (7) : 1026-1032 (1995)

Kessler RC, Crum RM, Warner LA, Nelson CB, Schulenberg J \& Anthony JC: "Lifetime co-occurrence of DSM-III-R alcohol abuse and dependence with other psychiatric disorders in the National Comorbidity Survey" - In Archives of General Psychiatry, 54 (4) : 313-321 (1997)

Khantzian EJ: "The self-medication hypothesis of addictive disorders: Focus on heroin and cocaine dependence" - In American Journal of Psychiatry, 142 (11) : 1259-1264 (1985)

Khantzian EJ: "The self-medication hypothesis of substance use disorders: A reconsideration and recent applications" - In Harvard Review of Psychiatry, 4 (5) : 231-244 (1997)

Klassen D \& O'Connor WA: "Predicting violence in schizophrenic and non-schizophrenic patients: A prospective study" - In Journal of Community Psychology, 16 (2) : 217-227 (1988)

Lehman AF, Myers CP, Dixon LB \& Johnson JL: "Detection of substance use disorders among psychiatric inpatients" - In Journal of Nervous \& Mental Disease, 184 (4) : 228-233 (1996)

Lehman AF, Myers CP, Dixon LB \& Johnson JL: "Defining subgroups of dual diagnosis patients for service planning" - In Hospital \& Community Psychiatry, 45 (6) : 556-561 (1994) 
Link BG \& Stueve A: "Psychotic symptoms and the violent/illegal behavior of mental patients compared to community controls" - In J Monahan \& HJ Steadman: Violence and mental disorder: Developments in risk assessment, The John D and Catherine T MacArthur Foundation series on mental health and development : 137-159 (1994)

Link BG, Stueve A \& Phelan J: "Psychotic symptoms and violent behaviors: probing the components of "threat/control-override" symptoms" - In Social Psychiatry and Psychiatric Epidemiology, 33, S55-S60 (1998)

Maneros A, Deister A \& Rohde A: "Comparison of long-term outcome of schizophrenic, affective, and schizoaffective disorders" - In British Journal of Psychiatry, 161 : 44-51 (1992)

McGurk SR \& Meltzer HY: "The role of cognition in vocational functioning in schizophrenia" - In Schizophrenia Research, 45 : 175-184 (2000)

McGurk SR, Mueser KT, Harvey PD, Marder J \& LaPuglia R: "Cognitive and clinical predictors of work outcomes in clients with schizophrenia" - In Psychiatric Services, $54: 1129-1135$ (2003)

Melle I, Friis S, Hauff E \& Vaglum P: "Patients with schizophrenia after the acute ward: Seven years service utilization and clinical course" - In Nordic Journal of Psychiatry, 54 (1) : 47-54 (2000)

Mercier C \& Beaucage B : Toxicomanie et problèmes sévères de santé mentale : Recension des écrits et état de la situation pour le Québec - Rapport au ministre de la Santé et des Services sociaux. Comité permanent de lutte à la toxicomanie, Gouvernement du Québec (1997)

Miner CR, Rosenthal RN, Hellerstein DJ \& Muenz LR: "Prediction compliance with outpatient referral in patients with schizophrenia and psychoactive substance use disorders" - In Archives of General Psychiatry, 54 (8) : 706-712 (1997)

Monahan J \& Appelbaum P: "Reducing violence risk : diagnostically based clues from the MacArthur Violence among the Mentally Ill" - In S. Hodgins (Ed) : Effective prevention of crime and violence among the mentally ill. The Netherlands ; Kluwer Academic Publishers (2000)

Mueser T, Bellack AS \& Blanchard JJ: "Comorbidity of schizophrenia and substance abuse: Implications for treatment" - In Journal of Consulting \& Clinical Psychology, 60 (6) : 845-856 (1992)

Mueser KT, Drake RE, Ackerson TH, Alterman AI, et al.: "Antisocial personality disorder, conduct disorder, and substance abuse in schizophrenia" - In Journal of Abnormal Psychology, 106 (3) : 473-477 (1997)

Mueser KT, Drake RE \& Wallach MA: "Dual diagnosis: A review of etiological theories" - In Addictive Behaviors, 23 (6) : 717-734 (1998)

Mueser KT, Salyers MP \& Mueser PR: “A prospective analysis of work in schizophrenia” - In Schizophrenia Bulletin, 27 : 281-296 (2001)

Mulkern V \& Manderscheid R: "Characteristics of community support program clients in 1980 and 1984" - In Hospital and Community Psychiatry, 40 : 165-172 (1989)

Nadeau L : «Lorsque le tout est plus grand que la somme de ses parties : la co-occurrence de la toxicomanie et des autres troubles mentaux. Éditorial »- In Santé mentale au Québec, XXVI (2) : 2-21 (2001)

Noordsy DL, Drake RE, Teague GB, Osher FC, et al.: "Subjective experiences related to alcohol use among schizophrenics" - In Journal of Nervous \& Mental Disease, 179 (7) : 410-414 (1991)

Noordsy DL, O'Keefe C, Mueser KT \& Xie HY: "Six-month outcomes for patients who switched to olanzapin treatment” - In Psychiatric Services 52 : 501-507 (2001) 
Osher FC \& Drake RE: "Reversing a history of unmet needs: Approaches to care for persons with co-occurring addictive and mental disorders" - In American Journal of Orthopsychiatry, 66 (1) : 4-11 (1996)

Owen RR, Fischer EP, Booth BM \& Cuffel BJ: "Medication noncompliance and substance abuse among patients with schizophrenia" - In Psychiatric Services, 47 (8) : 853-858 (1996)

Parent I \& Brochu S: "Drug/Crime Pathways Among Cocaine Users" - In S Brochu, C Da Agra \& M-M Cousineau: Drugs and Crime Deviant Pathways. Montréal, Centre international de criminologie (2002)

Pernanen K, Cousineau M-M, Brochu S \& Sun F : Proportions des crimes associés à l'alcool et aux autres drogues au Canada - Rapport présenté au Centre canadien de lutte contre l'alcoolisme et les toxicomanies (2002)

Potvin S, Stip E \& Roy JY: "Schizophrenia and addiction: An evaluation of the selfmedication hypothesis" - In Encephale, 29 (3) : 193-203 (2003)

Pristach CA \& Smith CM: "Self-reported effects of alcohol use on symptoms of schizophrenia" - In Psychiatric Services, 47 (4) : 421-423 (1996)

Regier DA, Farmer ME, Rae DS, Locke BZ, Keith SJ, Judd LL, \& Goodwin FK: "Comorbidity of mental disorders with alcohol and other drug abuse : Results from the Epidemiologic Catchment Area (ECA) study" - In Journal of the American Medical Association, 264 : 2511-2518 (1990)

Rice ME \& Harris GT: "Psychopathy, schizophrenia, alcohol abuse, and violent recidivism" - In International Journal of Law \& Psychiatry, 18 (3) : 333-342 (1995)

Robins LN \& Regier DA: Psychiatric disorders in America - New York, The Free Press (1990)

Robins LN, Tipp J \& Przybeck T: "Antisocial personality" - In LN Robins et DA Regier: Psychiatric disorders in America: The Epidemiologic Catchment Area Study (p. 285-290), New York, Free Press (1991)

Sajatovic M, Valenstein M, Blow FC, Ganoczy D \& Ignacio RV: "Treatment adherence with antipsychotic medications in bipolar disorder" - In Bipolar Disorders. 8 (3) : 232-41 (2006)

Santé Canada: Meilleures pratiques: Troubles concomitants de santé mentale et d'alcolisme et de toxicomanie - Santé Canada (2002)

Scheller-Gilkey G, Woolwine BJ, Cooper I, Gay O, Moynes KA \& Miller AH: "Relationship of clinical symptoms and substance use in schizophrenia patients on conventional versus atypical antipsychotics" - In American Journal of Drug \& Alcohol Abuse, 29 (3) : 553-566 (2003)

Shaner A, Eckman TA, Roberts LJ, Wilkins JN, et al.: "Disability income, cocaine use, and repeated hospitalization among schizophrenic cocaine abusers: A governmentsponsored revolving door" - In New England Journal of Medicine, 333 (12) : 777783 (1995)

Smelson DA, Losonczy MF, Kilker C, Starosta A, Kind J, Williams J \& Ziedonis D: "An analysis of cue reactivity among persons with and without schizophrenia who are addicted to cocaine" - In Psychiatric Services, 53 (12) : 1612-1616 (2002)

Smelson DA, Losonczy MF, Davis CW, Kaune M, Williams J \& Ziedonis D: "Risperidone decreases craving and relapses in individuals with schizophrenia and cocaine dependence" - In Canadian Journal of Psychiatry. 47 : 671-675 (2002) 
Schneeberger P : Profil de consummation de substances psychoactives chez les personnes judiciarisées - Rapport 1, Centre international de criminologie comparée pour le comité Toxico-Justice (1995)

Steadman HJ, Mulvey EP, Monahan J, Robbins PC, Appelbaum PS, Grisso T, Roth LH \& Silver E: "Violence by people discharged from acute psychiatric inpatient facilities and by others in the same neighborhoods" - In Archives of General Psychiatry, 55 (5) : 393-401 (1998)

Stuyt EB, Sajbel TA \& Allen MH: "Differing effects of antipsychotic medications on substance abuse treatment patients with co-occurring psychotic and substance abuse disorders" - In American Journal on Addictions. 15 (2) : 166-73 (2006)

Swanson JW, Holzer CE, Ganju VK \& Jono RT: "Violence and psychiatric disorder in the community: Evidence from the Epidemiologic Catchment Area surveys" - In Hospital \& Community Psychiatry, 41 (7) : 761-770 (1990)

Swanson J.W., Borum R., Swartz M.S., \& Monahan J: "Psychotic symptoms and disorders and the risk of violent behaviour in the community" - In Criminal Behaviour \& Mental Health, 6 (4) : 309-329 (1996)

Swanson JW, Swartz MS, Essock SM, Osher FC, Wagner HR, Goodman LA, Rosenberg SD \& Meador KG: "The social-environmental context of violent behavior in persons treated for severe mental illness" - In American Journal of Public Health, 92 (9) : 1523-1531 (2002)

Swartz MS, Swanson JW, Hiday VA, Borum R, Wagner R \& Burns BJ: "Taking the wrong drugs: The role of substance abuse and medication noncompliance in violence among severely mentally ill individuals" - In Social Psychiatry \& Psychiatric Epidemiology, 33 (Suppl 1) : 75-80 (1998)

Swartz MS, Swanson JW, Hiday VA, Borum R, Wagner R \& Burns BJ: "Violence and severe mental illness: The effects of substance abuse and nonadherence to medication" - In American Journal of Psychiatry, 55 (2) : 226-231 (1998)

Talamo A, Cebtirrino F, Tondo L, Dimitri A, Hennen J, Baldessarini RJ: "Comorbid substance-use in schizophrenia: Relation to positive and negative symptoms" - In Schizophrenia Research, $86: 251-255$ (2006)

Tiihonen J, Isohanni M, Rasanen P, Koiranen M \& Moring J: "Specific major mental disorders and criminality: A 26-year prospective study of the 1996 Northern Finland Birth Cohort" - In American Journal of Psychiatry, 54 (6) : 840-845 (1997)

Valenstein M, Ganoczy D, McCarthy JF, Myra Kim H, Lee TA \& Blow FC: “Antispychotic adherence over time among patients receiving treatment for schizophrenia: a retrospective review" - In Journal of Clinical Psychiatry, 67 (10) : 1542-50 (2006)

Voruganti LNP, Heslegrave RJ \& Awad AG: "Neuroleptic dysphoria may be the missing link between schizophrenia and substance abuse" - In Journal of Nervous \& Mental Disease, 185 (7) : 463-465 (1997)

Warner R, Taylor D, Wright J, Sloat A, et al.: "Substance use among the mentally ill: Prevalence, reasons for use, and effects on illness" - In American Journal of Orthopsychiatry, 64 (1) : 30-39 (1994)

Ziedonis DM \& Trudeau K: "Motivation to quit using substances among individuals with schizophrenia: implications for a motivation-based treatment model" - In Schizophrenia Bulletin, 23 (2) : 229-38 (1997)

Zimmet SV, Strous RD, Burgess ES, Kohnstamm S \& Green AI: "Effect of clozapine on substance use in patients with schizophrenia and schizoaffective disorder: A retrospective study" - In Journal of Clinical Psychopharmacology, 20 (1) : 94-98 (2000) 